\title{
Laser transfer of sol-gel ferroelectric thin films using an ITO release layer
}

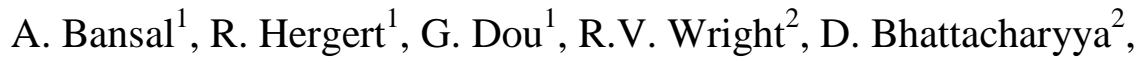 \\ P.B. Kirby ${ }^{2}$, E.M. Yeatman ${ }^{1}$, A.S. Holmes ${ }^{1 *}$
}

1. Department of Electrical \& Electronic Engineering, Imperial College, Exhibition Road, London, SW7 2AZ, UK

2. Microsystems \& Nanotechnology Centre, Materials Department, Cranfield University, Cranfield, Bedfordshire, MK43 0AL, UK

* Corresponding author email: a.holmes@imperial.ac.uk

\begin{abstract}
A new laser transfer process is reported which allows damage-free transfer of ferroelectric thin films from a growth substrate directly to a target substrate. The thin film ferroelectric material is deposited on a fused silica growth substrate with a sacrificial release layer of ITO (indium tin oxide). Regions of the film that are to be transferred are then selectively metallised, and bonded to the target substrate. Separation from the growth substrate is achieved by laser ablation of the ITO release layer by a single pulse from a KrF excimer laser, with the laser light being incident through the growth substrate. The residual ITO on the transferred ferroelectric layer is electrically conducting, and may be suitable for incorporation into the final device, depending on the application. The new process has been demonstrated for $500 \mathrm{~nm}$-thick layers of sol-gel PZT which were thermosonically bonded to a silicon target substrate prior to laser release. The transferred films show ferroelectric behaviour and have a slightly reduced permittivity compared to the as-deposited material.
\end{abstract}




\section{Introduction}

Ferroelectric materials such as lead zirconate titanate (PZT) have potential applications in a wide range of miniaturised devices, including RF components (e.g. fixed capacitors, varactors and resonators), ferroelectric memories, and piezoelectric sensors and actuators [1-3]. However, the high processing temperatures required to produce dense and fully crystallised ferroelectric layers can make integration of these materials into such devices challenging. For example, thin film processing methods typically involve annealing temperatures in the range 500-700 ${ }^{\circ} \mathrm{C}$. This precludes fully monolithic fabrication on low-temperature (e.g. polymer) substrates which are of increasing interest in consumer electronics. Even with traditional substrate materials such as silicon the design possibilities are reduced because the ferroelectric film has to be deposited at an early stage before other materials are introduced. These compatibility issues are even more severe for thick films produced by tape-casting or screen printing, where sintering temperatures in the range $800-1000{ }^{\circ} \mathrm{C}$ are typical.

The difficulties associated with fully monolithic integration can be avoided by forming the ferroelectric film on a high-temperature growth substrate and then transferring it to a second 'target' substrate where the rest of the device fabrication will take place. This kind of transfer can be achieved by bonding the film to the target substrate and then removing the growth substrate by some combination of mechanical grinding and chemical etching [4]. However, this approach is laborious and wasteful of growth substrate material. Another possibility is to use a growth substrate with a thin sacrificial layer, for example a metal oxide, which can be selectively etched away to release the film. A third option, which avoids the use of any wet chemicals during release, is to use laser transfer processing (LTP), also referred to as laser lift-off (LLO). Here the film is released from the growth substrate by a pulse of laser radiation incident through the substrate. The laser wavelength is typically in the ultra-violet (UV), and is chosen such that the substrate is highly transmissive while the film to be transferred is strongly absorbing. The incident laser energy is absorbed in a thin layer of the film adjacent to the interface with the growth substrate, causing delamination to occur. The delamination process is generally attributed at least partly to ablative decomposition of the film, although in principle it could result purely from thermally induced shear stresses at the interface. 
Laser transfer for ferroelectric films was first demonstrated by Tsakalakos et al. who applied it to lanthanum-modified PZT thin films produced by pulsed laser deposition on $\mathrm{MgO}$ (magnesium oxide) substrates [5,6]. The $1.4 \mu \mathrm{m}$-thick films were first bonded to a stainless steel foil target using palladium-indium bonding. The same group have also demonstrated laser transfer for thin films of unmodified PZT. Other groups have applied laser transfer to thin and thick film PZT [7,8], and to various other ferroelectric materials in thin or thick film form, including barium strontium titanate, bismuth titanate, lanthanum-modified bismuth titanate and bismuth ferrite-lead titanate $[8,9]$. In most of the work to date, silver-loaded epoxy resin has been used to bond the film to the target substrate prior to laser release.

Laser transfer by direct absorption in the ferroelectric film provides an elegant, simple and fast method for release from the growth substrate. However, this approach does have one significant drawback: it leaves a laser-damaged layer at the surface of the released film in which the ferroelectric properties are severely degraded. This layer results from the transient heating induced by the laser pulse, and is typically of the order of $100 \mathrm{~nm}$ thick for films released by excimer laser. The damaged layer can occupy a significant fraction of the total film thickness, in which case the ferroelectric and dielectric behaviour of the film as a whole will be compromised. Previous work has shown that the damage layer can be removed, and the electrical properties recovered, by ion milling [5,6] or by polishing [8]. However, the first of these approaches is too slow to apply over large areas, while the second may not be sufficiently controllable for thin films.

This paper reports on a modified laser transfer process in which the laser energy is absorbed in a sacrificial release layer of indium tin oxide (ITO) that is deposited on the growth substrate prior to deposition of the ferroelectric film. In this way, any laser damage during release occurs in the ITO layer which can subsequently be removed by wet or dry etching. Alternatively, since ITO is electrically conducting, it may be appropriate to retain the ITO as a top electrode in low-frequency applications where highly conductive electrodes are not essential. Figure 1 shows an overview of the process, including both the film preparation and laser transfer. The process has been demonstrated for PZT films deposited on fused silica substrates by sol-gel processing and released using a $\mathrm{KrF}$ excimer laser (248 nm wavelength). Thermosonic bonding was used to attach the films to silicon substrates prior to laser release. 
Laser transfer using release layers has been applied previously to micromachined components fabricated directly on silica wafers [10], and to components fabricated on silicon wafers and transferred to glass carriers by bonding and substrate removal by grinding and etching [11,12]. It has also been used to release electronic components from glass carriers [13]. Polymer release layers have been used in all such processes to date. Polymers are particularly attractive because they combine strong UV optical absorption with relatively poor thermal conductivity and low decomposition temperature, resulting in a low ablation threshold and hence a low fluence (energy per unit area) threshold for release. Unfortunately, however, polymer materials cannot be used as release layers on growth substrates for ferroelectric films, because of the high processing temperatures involved. Certain refractory metals could be used, but the much higher thermal conductivity, coupled with the high ablation threshold, would increase the likelihood of thermally-induced damage in the adjacent ferroelectric film. The high ablation threshold would also probably make the release process relatively violent and likely to produce mechanical damage. ITO was chosen for the present work because it combines a high melting point (ca $2000 \mathrm{~K}$ ) with strong optical absorption and a low ablation threshold in the UV. The ablation properties of ITO and other transparent conductive oxides have been studied extensively because of their potential applications in solar panels and displays. The ablation threshold in an air ambient is around $100 \mathrm{~mJ} / \mathrm{cm}^{2}$ at $248 \mathrm{~nm}$ wavelength [14] which is comparable to that of many polymer materials.

\section{Experimental}

\section{Film preparation}

Figure 1a outlines the process used to produce thin PZT films for bonding and laser transfer. The growth substrates were prepared by depositing a $0.5 \mu \mathrm{m}$-thick layer of ITO onto a 4"diameter, $500 \mu \mathrm{m}$-thick fused silica wafer. The ITO was deposited by DC sputtering from a powdered ITO target (90/10 composition) in an argon plasma containing $0.5 \%$ oxygen. The resulting films were $\sim 80 \%$ transmissive at $500 \mathrm{~nm}$ wavelength and had a DC sheet resistance of $\sim 100 \Omega / \square$.

The sol-gel PZT films were deposited using previously reported methods [15]. First, a seed layer comprising $10 \mathrm{~nm}$ of titanium (Ti) for adhesion and $100 \mathrm{~nm}$ of platinum $(\mathrm{Pt})$ was deposited over the ITO. Pt is commonly used as a bottom electrode for sol-gel PZT films deposited on oxidised silicon because it promotes the formation of the desired perovskite 
phase of the PZT. The PZT film was built up by a sequence of spin-coating, pre-firing and annealing steps. The thickness of each spin-coated layer was around $70 \mathrm{~nm}$ after annealing, and seven layers were deposited to produce a film with a nominal thickness of $0.5 \mu \mathrm{m}$. Each layer was pre-fired at $200{ }^{\circ} \mathrm{C}$ for 2 minutes for pyrolysis, and then annealed at $530{ }^{\circ} \mathrm{C}$ for 5 minutes for crystallisation.

Following the PZT deposition, regions of the PZT layer that were to be transferred were selectively metallised by photolithography and electroplating. A sputtered chrome/copper layer ( $20 \mathrm{~nm} \mathrm{Cr}$ followed by $60 \mathrm{~nm} \mathrm{Cu}$ ) was deposited to act as an electroplating seed layer. A plating mould with square apertures of various sizes up to $800 \times 800 \mu \mathrm{m}^{2}$ was then formed in a $10 \mu \mathrm{m}$-thick layer of AZ9260 photoresist, and nickel and gold were electroplated into the apertures to depths of $5 \mu \mathrm{m}$ and $1 \mu \mathrm{m}$ respectively. Finally the photoresist was stripped to leave a series of metallised islands or "pads". The metallisation served a dual role of providing a bondable top surface for the PZT pads and increasing the mechanical robustness of the PZT layer during the release process. In earlier laser release experiments it was found that un-metallised thin films of PZT tend to fracture upon release from the growth substrate.

In the final preparation step, the material surrounding each metallised PZT pad was removed by laser machining down through all the deposited layers $(\mathrm{Cu} / \mathrm{Cr} / \mathrm{PZT} / \mathrm{Pt} / \mathrm{Ti} / \mathrm{ITO})$, stopping at the silica substrate. This was done using the $\mathrm{KrF}$ excimer laser in direct-write mode, with a laser spot formed by projecting a mask aperture. Chemical etching could equally well have been used here, but laser machining was chosen as it allowed all layers to be removed in a single operation. A layer of photoresist was spun over the wafer prior to laser machining to protect the gold-metallised areas from ablation debris. This protective layer was later removed, together with any residual ablation debris, using acetone.

\section{Thermosonic bonding}

Direct gold-gold thermosonic (TS) bonding was used to attach the metallised PZT pads to target substrates prior to laser release (Figure 1b). TS bonding is widely used in electronic packaging, both for wire-bonding and flip-chip attachment [16]. It has the advantages that it does not require any additional joining materials or fluxes, and the resulting assembly is free from organic materials that might degrade over time. The electrical performance is also better than that obtained by most other joining methods. On the other hand, TS bonding is generally 
limited to device or die level because it is difficult to distribute the ultrasonic energy and bonding force with sufficient uniformity over large areas.

TS bonding was carried out using a custom bonder developed for thermosonic flip chip assembly [17]. Each PZT pad was bonded to the centre of a gold-metallised silicon die. The $3 \times 3 \mathrm{~mm}^{2}$ dies were fabricated by electroplating gold onto a silicon wafer and then dicing the wafer by deep reactive ion etching. Bonding was carried out with the growth wafer mounted on the heated platform of the TS bonder, and the silicon die held in the ultrasonic bond tool. Nominal values for the bonder parameters were: a platform temperature of $200^{\circ} \mathrm{C}$, a bonding force of $0.015 \mathrm{gf} / \mathrm{mm}^{2}$, and an ultrasonic power of $20 \mathrm{~W}$ applied for $500 \mathrm{msec}$.

\section{Laser release}

Laser release experiments were carried out using an Exitech laser workstation equipped with an LPX 220i excimer laser (248 $\mathrm{nm}$ wavelength, $30 \mathrm{~ns}$ pulse duration). The system includes a beam homogeniser and an in-line attenuator ensuring uniform illumination and a well-defined fluence at the sample. The illuminated area at the sample plane was defined by projecting a mask aperture of known size using a $4 \mathrm{X}$ reduction lens. Fluence measurements were made by measuring the total pulse energy beyond the focal plane, and dividing by the illuminated area.

For release experiments, the growth wafer was suspended upside down over a receiving substrate with a small gap between the two. The laser beam was incident from above so that the released die, with attached PZT film, fell onto the receiving substrate.

\section{Analysis}

The structure of the as-deposited PZT films was analysed by XRD, using a Siemens D5005 $\mathrm{X}$-ray diffractometer configured for $\mathrm{CuK}_{\alpha}$ radiation. Electrical characterisation was also carried out, both on as-deposited films and on transferred films. This included small-signal AC capacitance and loss measurements, made using a Wayne-Kerr 6425 Precision Component Analyser, and PE loop measurements made using a Radiant Technologies RT66a Ferroelectric Test System. Cr/Au dots were sputtered onto the as-deposited films to form top electrodes, with the underlying Ti/Pt layer being used as a bottom electrode. The transferred films were tested using the residual ITO (with underlying Ti/Pt) as the top electrode, with connection to the underside being made via the gold surface on the target die. 


\section{Results and Discussion}

A key requirement for any process of the type reported here is that it must be possible to form properly crystallised ferroelectric films on the release layer. Figure 2 shows a typical XRD measurement for a PZT film deposited on Pt/Ti/ITO/silica. The peaks attributed to PZT are all for the perovskite phase, confirming that crystallisation has been achieved. However, many different perovskite orientations are represented, indicating that the film is not predominantly (111)-oriented as can be obtained for films deposited on a $\mathrm{Pt} / \mathrm{Ti} / \mathrm{SiO}_{2} / \mathrm{silicon}$ substrate. A likely explanation for this is that the surface roughness of the sputtered ITO is disrupting the orientation of the Pt layer and hence preventing proper alignment of the PZT. If so it may be possible achieve lower surface roughness, and more highly oriented PZT, by annealing the sputtered ITO films in oxygen, or by using ITO films grown by PLD (pulsed laser deposition).

The XRD plot also shows several peaks for ITO, one of which (at $2 \theta=30^{\circ}$ ) coincides with the position of the unwanted pyrochlore phase of PZT. Consequently it is not possible to conclude from the XRD plot alone that full crystallisation into the perovskite phase has taken place. However, capacitance measurements on the as-deposited films yielded dielectric constants in the range $370-450$ at low frequency $(1 \mathrm{kHz})$, which is consistent with full crystallisation; much lower values would be expected for films containing pyrochlore.

Release experiments were carried out over a wide range of fluences, from $375 \mathrm{~mJ} / \mathrm{cm}^{2}$ up to $1125 \mathrm{~mJ} / \mathrm{cm}^{2}$. At lower fluence levels, typically up to around $450 \mathrm{~mJ} / \mathrm{cm}^{2}$, a single pulse did not lead to any release. Above this level there was a narrow intermediate fluence range where a single pulse tended to produce only partial release, and if further pulses were applied the film would release fully but with some damage. At fluence levels above about $500 \mathrm{~mJ} / \mathrm{cm}^{2}$ release was consistently achieved with a single laser pulse. This value is near the middle of the range of fluences used previously for excimer laser transfer of ferroelectric films by direct absorption. For example, refs [5-9] report fluences over the range 250 to $700 \mathrm{~mJ} / \mathrm{cm}^{2}$. The fluence levels required for excimer laser transfer with organic release layers are typically somewhat lower, in the range 100 to $300 \mathrm{~mJ} / \mathrm{cm}^{2}$ (see e.g. [10]). 
Figure 3 shows an optical micrograph of a $600 \times 600 \mu \mathrm{m}^{2}$ pad released with a fluence of 525 $\mathrm{mJ} / \mathrm{cm}^{2}$. Some discoloration of the release layer has occurred, which is typical for excimer laser exposure of ITO [14]. A small area of the ITO has also been lost at the release step, exposing the underlying Ti/Pt. This is an artefact of the way the material was removed from around the pads; some clearance was left between the laser spot and the edge of the pad, resulting in a fillet of unsupported material that tends to fracture during laser release. This problem, and the generally poor edge quality of the pad, could be improved by refining the laser machining process or replacing it by chemical etching. Finally, several probe marks left behind by electrical testing can be seen near the centre of the pad.

Figure 4 shows a comparison between measured PE loops for as-deposited and laser transferred films. Both films exhibit shifts along the field axis but in opposite directions: +25 $\mathrm{kV} / \mathrm{cm}$ and $-85 \mathrm{kV} / \mathrm{cm}$ respectively. Such asymmetric switching indicates the presence of an internal electric field and this also accounts for the observed preference for a particular polarisation direction, evidenced by the difference in the positive and negative remanent polarisations ( $\mathrm{Pr}+$ and $\mathrm{Pr}-)$. Although electric fields can exist in the bulk of films, in our case it is most likely that the operative ones are located at the electrode-PZT interfaces and that the shift is linked to the use of different materials for the top and bottom electrodes [18]. The relatively large shift in the case of the laser transferred film is believed to be due to some artefact of processing, such as the sputter cleaning process prior to deposition of the electroplating seed layer, which was not used for the 'as deposited' measurements. The coercive field after removal of the field axis shift is $(\mathrm{Ec}+-\mathrm{Ec}-) / 2=115 \mathrm{kV} / \mathrm{cm}$ which is slightly lower than the value of $127 \mathrm{kV} / \mathrm{cm}$ reported previously for PZT films formed on $\mathrm{Si}$ substrates by a similar sol-gel process [15]. In contrast, thin PZT films formed on sapphire substrates by higher temperature sol-gel processing, and transferred by direct absorption in the PZT, showed a significant increase in coercive field (from $50 \mathrm{kV} / \mathrm{cm}$ to $150 \mathrm{kV} / \mathrm{cm}$ ) due to the formation of a low-permittivity damage layer [8,9].

The remanent polarisation values for the transferred film are $\operatorname{Pr}+=32 \mu \mathrm{C} / \mathrm{cm}^{2}$ and $\operatorname{Pr}-=-17$ $\mu \mathrm{C} / \mathrm{cm}^{2}$, compared to $\operatorname{Pr}+=9 \mu \mathrm{C} / \mathrm{cm}^{2}$ and $\operatorname{Pr}-=-12 \mu \mathrm{C} / \mathrm{cm}^{2}$ for the as-deposited film. The remanent polarisation values in the pre- transferred film are significantly lower than the value of $\operatorname{Pr}=24 \mu \mathrm{C} / \mathrm{cm}^{2}$ reported in [15] for films formed on Si, probably because of the larger thermal mismatch between PZT and glass compared to that of PZT and Si and consequently 
larger tensile stress [19]. Furthermore the higher, close to normal, values observed for the transferred film are believed to be the result of stress relaxation in the film following release from the growth substrate. For comparison, values of $\operatorname{Pr}=24 \mu \mathrm{C} / \mathrm{cm}^{2}$ and $\operatorname{Pr} \sim 30 \mu \mathrm{C} / \mathrm{cm}^{2}$ were reported in [8] and [9] respectively for sol-gel PZT films transferred from sapphire substrates without a release layer.

Figure 5 shows dielectric constant and loss tangent measurements for the same transferred film. The dielectric constant, which varies from 319 at $1 \mathrm{kHz}$ to 309 at $300 \mathrm{kHz}$, is reduced compared to that of the as-deposited film, though not significantly. The film shows low loss $(0.005)$ at $1 \mathrm{kHz}$, but the loss increases to 0.09 at $300 \mathrm{kHz}$. It is believed that the increase may be due to the series resistance of a laser-damaged layer at the top surface of the ITO, but this aspect requires further investigation.

\section{Conclusion}

A new laser transfer process for ferroelectric thin films has been demonstrated in which the laser energy is absorbed in an ITO release layer, thereby avoiding laser damage to the transferred film. To our knowledge this is the first report of thin film laser transfer directly from a high-temperature growth substrate using a release layer. The residual ITO on the transferred film can be removed by selective etching, although for some applications it may be appropriate to retain the ITO as a top electrode. The initial results presented here indicate that the process does not result in any significant degradation of the ferroelectric properties, although further analysis is required to establish conclusively the reasons for the changes that are observed, in particular the offsetting of the PE loop in the transferred material. The new process has been demonstrated only for sol-gel PZT films deposited on fused silica substrates, with thermosonic bonding to silicon target substrates. However, it has the potential to be combined with other film fabrication methods, bonding techniques, and growth/target substrate materials.

\section{Acknowledgement}

The authors gratefully acknowledge the financial support of the UK Engineering and Physical Sciences Research Council under the Flagship Project EP/D064805/1, "Integrated functional materials for system-in-package applications". 


\section{References}

1. N. McN. Alford, O.Yu. Buslov, V.N. Keis, A.B. Kozyrev, P.K. Petrov, A.Yu. Shimko, "Band-pass tunable ferroelectric filter based on unipolar dielectric resonators", Proc. $1^{\text {st }}$ European Wireless Technol. Conf., (2008), 282-285.

2. B. Lei, C. Li, D. Zhang, Q.F. Zhou, K.K. Shung, C. Zhou, "Nanowire transistors with ferroelectric gate dielectrics: enhanced performance and memory effects", Appl. Phys. Lett., 84(22), (2004), 4553-4555.

3. A. Safari, M. Allahverdi, E.K. Akdogan, "Solid freeform fabrication of piezoelectric sensors and actuators", J. Mat. Sci., 41, (2006), 177-198.

4. T. Riekkinen, T. Mattila, S. van Dijken, A. Lüker, Q. Zhang, P.B. Kirby, A.M. Sánchez, "Ferroelectric parallel-plate capacitors with copper electrodes for high-frequency applications", Appl. Phys. Lett., 91, 252902 (2007).

5. L. Tsakalakos, T. Sands, "Epitaxial ferroelectric $(\mathrm{Pb}, \mathrm{La})(\mathrm{Zr}, \mathrm{Ti}) \mathrm{O}_{3}$ thin films on stainless steel by excimer laser liftoff”, Appl. Phys. Lett., 76, (2000), 227-229.

6. L. Tsakalakos, T. Sands, E. Carleton, K.M. Yu, "Modification of ( $\mathrm{Pb}, \mathrm{La})(\mathrm{Zr}, \mathrm{Ti}) \mathrm{O}_{3}$ thin films during pulsed laser liftoff from MgO substrates”, J. Appl. Phys., 94(6), (2003), 4047-4052.

7. B. Xu, D. White, J. Zesch, A. Rodkin, S. Buhler, J. Fitch, K. Littau, "Characteristics of lead zirconate titanate ferroelectric thick films from a screen-printing laser transfer method”, Appl. Phys. Lett., 87(19), 192902 (2005).

8. C. James, T. Chakraborty, A. Brown, T. Comyn, R. Dorey, J. Harrington, A.J. Laister, R.E. Miles, C. Puchmark, B. Xu, W. Xiong, Q. Zhang, S.J. Milne, "Laser transfer processing and the integration of ferroelectric films", J. Mat. Sci., 44, (2009), 5325-5331.

9. T. Chakraborty, B. Xu, Q. Zhang, A.J. Bell, X. Bo, A. Chowdhury, C. James, C. Puchmark, J. Harrington, M. Khan, R.E. Miles, W. Xiong, S.J. Milne, "Laser transfer processing for the integration of thin and thick film ferroelectrics", Integrated Ferroelectrics, 106, (2009), 40-48.

10. A.S. Holmes, S.M. Saidam, "Sacrificial layer process with laser-driven release for batch assembly operations", J. Microelectromech. Syst., 7(4), (1998), 416-422.

11. R. Guerre, U. Drechsler, D. Jubin, M. Despont, "Selective transfer technology for microdevice distribution", J. Microelectromech. Syst., 17(1), (2008), 157-165.

12. R. Guerre, U. Drechsler, D. Bhattacharyya, P. Rantakari, R. Stutz, R.V. Wright, Z.D. Milosavljevic,, T. Vähä-Heikkilä, P.B. Kirby, M. Despont, "Wafer-level transfer technologies for PZT-based RF MEMS switches", J. Microelectromech. Syst., 19(3), (2010), 548-560.

13. C.B. Arnold, P. Serra, A. Piqué, "Laser direct-write techniques for printing complex materials", MRS Bulletin, 32, (2007), 23-31.

14. K. Lee, C. Lee, "The comparison of ITO ablation characteristics using KrF excimer and Nd:YAG laser", Proc. $2^{\text {nd }}$ Int. Symp. On Laser Prec. Microfab., SPIE vol. 4426, (2002), 260-263.

15. Q. Zhang, R.W. Whatmore, "Sol-gel PZT and Mn-doped PZT thin films for pyroelectric applications", J. Phys. D: Appl. Phys., 34, (2001), 2296-2301.

16. S.-Y. Choi, C.-S., Ti, M.-H. Park, S.-T. Chang, "New ultra-thin chip scale package (CSP) based on thermosonic flip-chip interconnection", Proc. $9^{\text {th }}$ Electronics Packaging Technology Conference, (2007), 892-896.

17. Gao S., Holmes A.S., "Thermosonic flip chip interconnection using electroplated copper column arrays", IEEE Trans. Adv. Packaging, 29(4), (2006), 725-734.

18. J. Lee, C.H. Choi. B.H. Park, T.W. Noh, J.K. Lee, "Built-in voltages and asymmetric polarization switching in $\mathrm{Pb}(\mathrm{Zr}, \mathrm{Ti}) \mathrm{O}_{3}$ thin film capacitors", Appl. Phys. Lett., 72(25), (1998), 3380-3382.

19. J.W. Lee, C.S. Park, M. Kim, H.-E. Kim, "Effects of residual stress on the electrical properties of PZT films", J. Am. Ceram. Soc., 90(4), (2007), 1077-1080. 


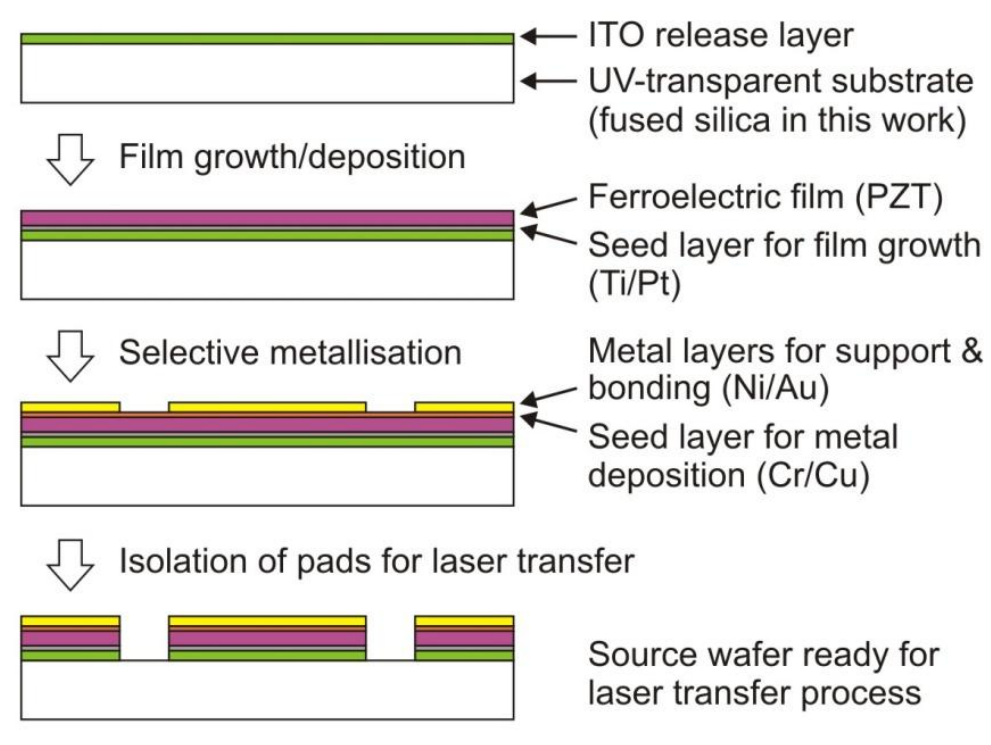

(a)

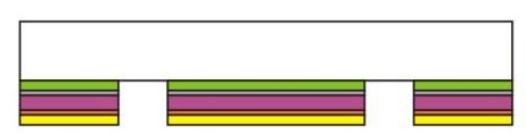

Source wafer

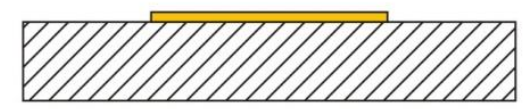

Target substrate with bonding layer

Alignment and bonding

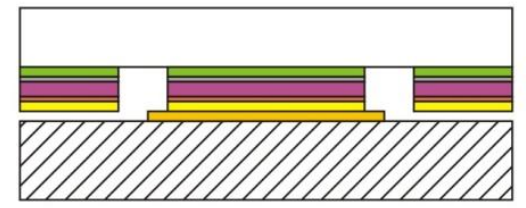

Laser exposure

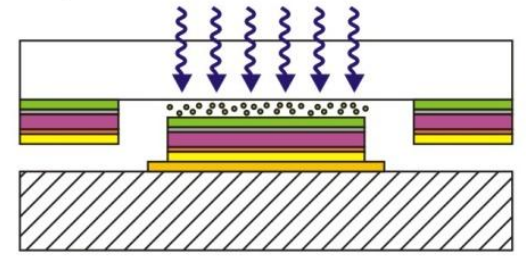

Removal of residual release layer (if necessary)

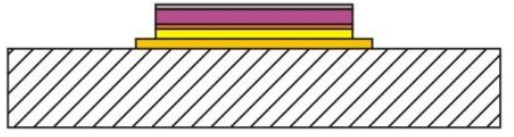

(b)

Figure 1: Outline of laser transfer process: (a) preparation of ferroelectric film on growth substrate with release layer; (b) bonding to target substrate and laser release from growth substrate. 


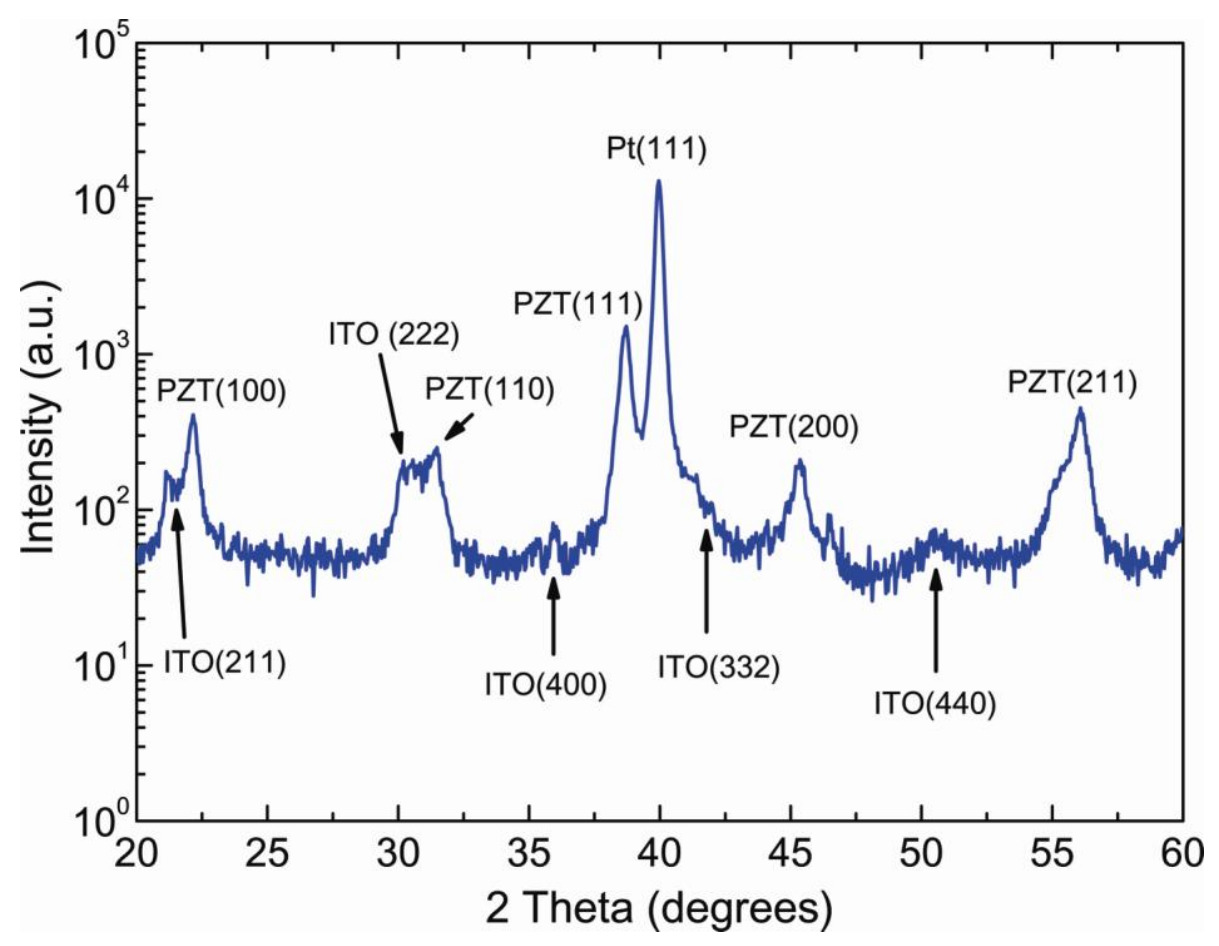

Figure 2: XRD plot for $0.5 \mu$ m-thick sol-gel PZT deposited on Pt/Ti/ITO/silica.

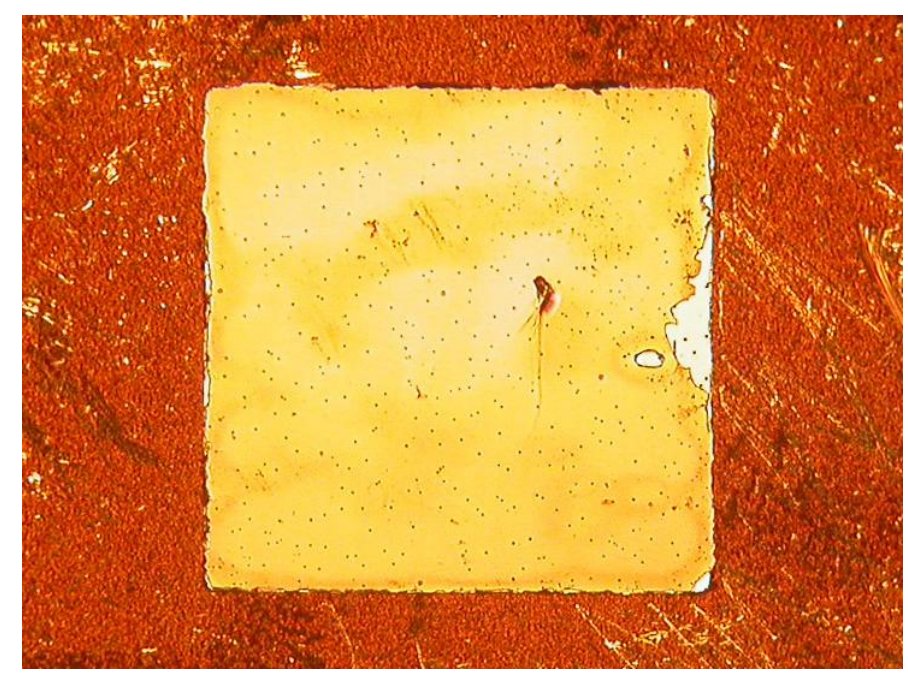

Figure 3: Optical micrograph showing a $600 \times 600 \mu \mathrm{m}^{2}, 0.5 \mu \mathrm{m}$-thick pad of PZT after thermosonic bonding and laser transfer to a gold-metallised silicon substrate. The lighter central region is residual ITO, with underlying Ti/Pt/PZT; surround is electroplated gold surface of target wafer. 


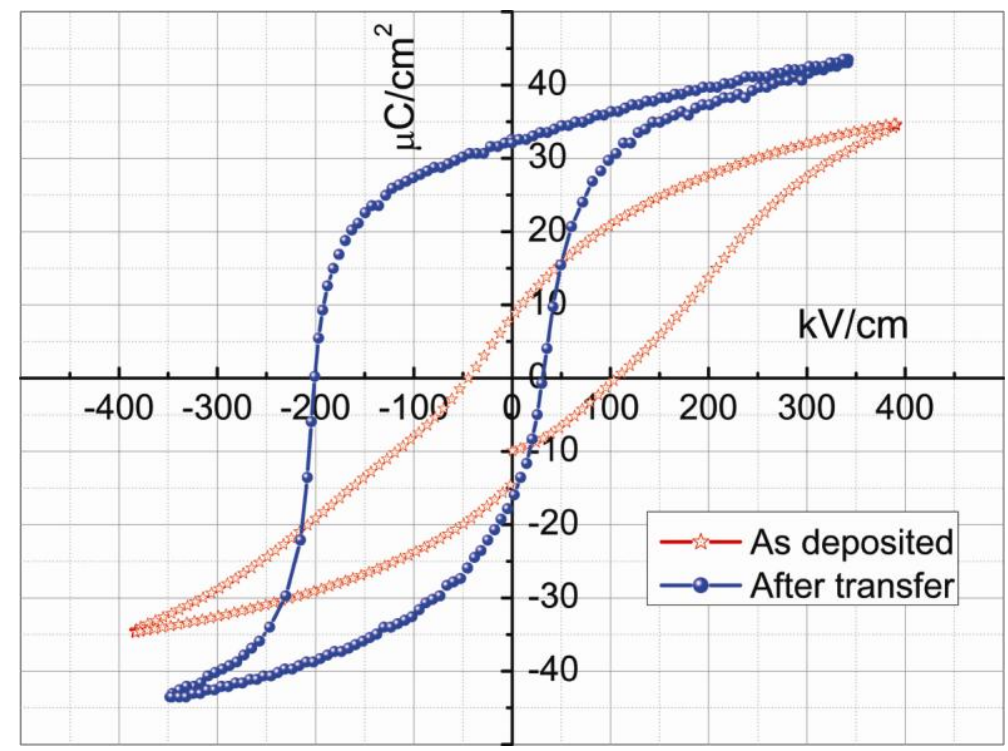

Figure 4: Measured P-E loops for $0.5 \mu \mathrm{m}$-thick sol-gel PZT films: (a) as deposited on $\mathrm{Pt} / \mathrm{Ti} / \mathrm{ITO} /$ silica, and (b) after thermosonic bonding and laser transfer to a gold-metallised silicon substrate.

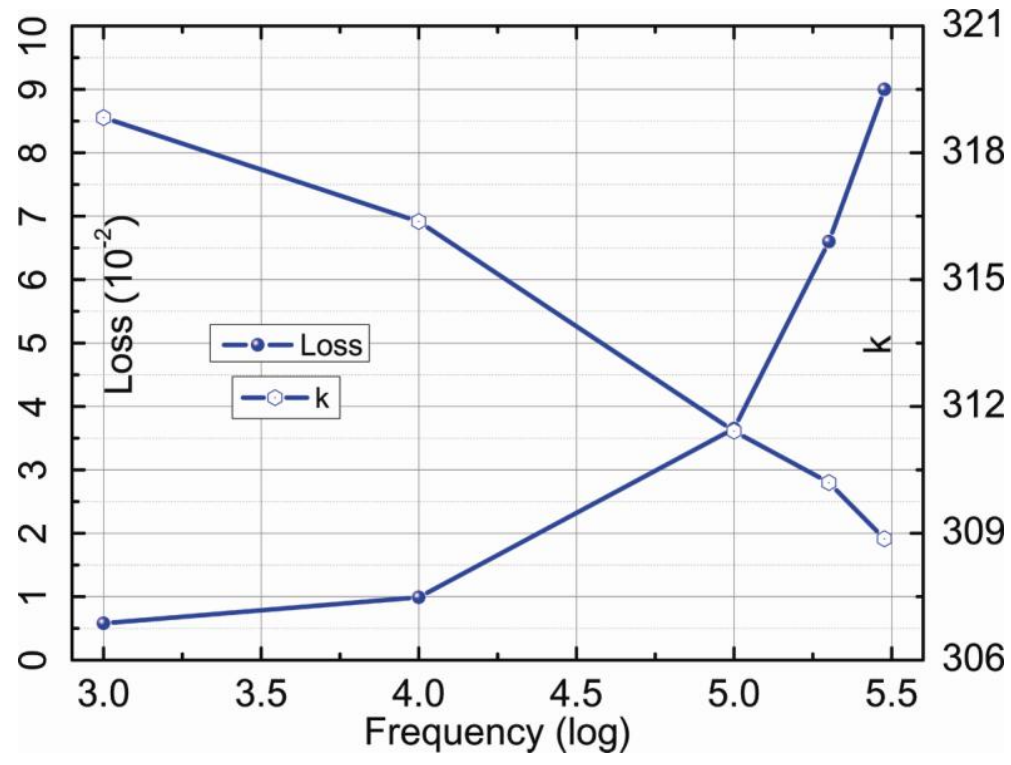

Figure 5: Measured frequency variations of dielectric constant and loss tangent for laser transferred film. Residual ITO, with underlying Ti/Pt, is used as the top electrode in these measurements. 